\title{
Prevention of Gallstone Formation After Gastrectomy
}

\author{
Henry A. Pitt, MD 1; Attila Nakeeb, MD 2 \\ 1 Department of Surgery, Lewis Katz School of Medicine at Temple University, Philadelphia, \\ Pennsylvania \\ 2 Department of Surgery, Indiana University School of Medicine, Indianapolis
}

The adverse effects of vagotomy and the association of gastric distension with biliary physiology have been appreciated for decades.1,2 More recently, risk factors for the development of gallstones after gastrectomy have been described.3,4 In addition, the first prospective, randomized, multicenter clinical trial of the use of ursodeoxycholic acid (UDCA) to prevent gallstones after gastrectomy was published 25 years ago. 5 However, that study and the vast majority of subsequent research have been performed for patients undergoing bariatric surgery. Thus, the study by Lee et al6 in this issue of JAMA Surgery is unique because it was performed for patients undergoing gastrectomy for gastric cancer.

The authors performed a multicenter, randomized, placebo-controlled clinical trial of daily UDCA, 300 mg, UCDA, $600 \mathrm{mg}$, or placebo for 52 weeks for 521 patients. Both doses of UDCA significantly reduced the incidence of gallstones after 1 year (with an odds ratio of 0.27 for $300 \mathrm{mg}$ and 0.20 for 600 $\mathrm{mg}$ ). However, when the UDCA was stopped, the incidence of gallstones rapidly increased from $5 \%$ to $10 \%$ in patients receiving $300 \mathrm{mg}$ of UDCA. Gallstone incidence also doubled from $17 \%$ to $35 \%$ in the patients who received placebo pills.

Unfortunately, this aspect of the study was not completely reported but suggests that follow-up for more than 1 year is important and that UCDA prophylaxis should not be stopped at 1 year. Another aspect of the study that was not reported was the demographic characteristics of the patients who developed gallstones after surgery. Did these patients have known risk factors, such as female sex, advanced age, or obesity? Were they more likely to have a distal gastrectomy or a postoperative complication or to receive adjuvant therapy? Data on these and other characteristics of the 40 patients who developed gallstones would be helpful in choosing the dose of UDCA and in designing future trials.

The other question raised by this Korean study is the generalizability to Western patients. 7 The median age of the study patients was 56 years, and two-thirds were men. The median body mass index (calculated as weight in kilograms divided by height in meters squared) was only 23, and more than $80 \%$ of the patients had stage I tumors. In comparison, Western patients with gastric cancer are older, more likely to be female and overweight, and are more likely to have more advanced tumors. Also, $84 \%$ of the tumors in the study by Lee et al 6 occurred in the distal stomach, $88 \%$ of the gastrectomies were performed laparoscopically, and $57 \%$ of the lymph node dissections were D-2. These tumor and operative factors also differ in Western countries. Although another randomized clinical trial for Western patients would be ideal, for multiple reasons, it would be difficult to perform. Thus, the authors' recommendations to give UDCA to prevent gallstones in patients undergoing gastrectomy for gastric cancer is appropriate. However, recommending a cholecystectomy for patients with a good cancer prognosis is another viable option.

This is the author's manuscript of the work published in final edited form as:

Pitt, H. A., \& Nakeeb, A. (2020). Prevention of Gallstone Formation After Gastrectomy. JAMA

Surgery, 155(8), 712-712. https://doi.org/10.1001/jamasurg.2020.1527 
Article Information

Corresponding Author: Henry A. Pitt, MD, PhD, Department of Surgery, Lewis Katz School of Medicine at Temple University, 3509 N Broad St, Boyer Pavillion, Philadelphia, PA 19140 (henry.pitt@tuhs.temple.edu).

Published Online: June 17, 2020. doi:10.1001/jamasurg.2020.1527

Conflict of Interest Disclosures: None reported. 


\section{References}

1. Pitt HA, Doty JE, Roslyn JJ, DenBesten L. The role of altered extrahepatic biliary function in the pathogenesis of gallstones after vagotomy. Surgery. 1981;90(2):418-425.

2. Webb TH, Lillemoe KD, Pitt HA. Gastrosphincter of Oddi reflex. Am J Surg. 1988;155(2):193-198. doi:10.1016/S0002-9610(88)80692-5

3. Kobayashi T, Hisanaga M, Kanehiro H, Yamada Y, Ko S, Nakajima Y. Analysis of risk factors for the development of gallstones after gastrectomy. Br J Surg. 2005;92(11):13991403. doi:10.1002/bjs.5117

4. Fukagawa T, Katai H, Saka M, Morita S, Sano T, Sasako M. Gallstone formation after gastric cancer surgery. J Gastrointest Surg. 2009;13(5):886-889. doi:10.1007/s11605-0090832-8

5. Sugerman HJ, Brewer WH, Shiffman ML, et al. A multicenter, placebo-controlled, randomized, double-blind, prospective trial of prophylactic ursodiol for the prevention of gallstone formation following gastric-bypass-induced rapid weight loss. Am J Surg. 1995;169(1):91-96. doi:10.1016/S0002-9610(99)80115-9

6. Lee SH, Jang DK, Yoo M-W, et al; Efficacy and Safety of DWJ1319 in the Prevention of Gallstone Formation after Gastrectomy in Patient with Gastric Cancer: A Multicenter, Randomized, Double-blind, Placebo-controlled Study (PEGASUS-D) Group. Efficacy and safety of ursodeoxycholic acid for the prevention of gallstone formation after gastrectomy in patients with gastric cancer: the PEGASUS-D randomized clinical trial. JAMA Surg. Published online June 17, 2020. doi:10.1001/jamasurg.2020.1501

7. Nakeeb A, Comuzzie AG, Martin L, et al. Gallstones: genetics versus environment. Ann Surg. 2002;235(6):842-849. doi:10.1097/00000658-200206000-00012 PubMedGoogle ScholarCrossref 